Warmem verbindet, so nimmt die luftige Substanz, die in jenen Gliedern ist, die Wärme auf, die sie nun der Seele in der logistischen Zelle gegenwärtig macht. Ähnlich ist es bei Kälte usw. usw.

Weiterhin gibt es eine Funktion der Seele, die Imagination (Einbildungskraft) genannt wird und eine Kraft der Seele ist, vermöge der wir die Gestalt und die Farbe eines gegenwärtigen Dings aufnehmen. Diese Funktion ist dazu im Menschen notwendig, damit nicht ein Ding der Vergessenheit anheimfällt. Gestalt und Farbe eines Menschen, den wir sehen, tragen wir nämlich durch die Imagination bei uns. Deshalb stellen wir ihn uns vor, wenn wir ihn wiedererkennen, und auch jemanden, den wir niemals wiedersehen. Aber im Verhältnis zur Ähnlichkeit eines Dings, das wir einmal gesehen haben. Daraus folgt, daß die Imagination aus dem Gesichtssinn geboren wird. Was nämlich stellen wir uns vor? Entweder stellen wir uns vor, was wir gegenwärtig sehen oder wir stellen uns ein Ding vor, das wir gesehen haben, und zwar im Verhältnis zu seiner Ähnlichkeit, von der gleichen Art, wie das Ding ist. Wie jener Tityrus bei V e r g i l sagt: Rom, das er niemals sah, stellte er sich ähnlich seiner Vaterstadt vor. Es sagt nämlich auch $A$ u g u s tin u s : Das rote Meer, das ich niemals sah, stellte ich mir nach der Ähnlichkeit eines anderen Meeres vor. Dieser Vorgang ist uns und den unvernünftigen Tieren gemeinsam. Daher kommt es, daß die unvernünftigen Tiere ihre Herren erkennen, den einen freundlich suchen, den andern fliehen können. Und dies stammt $n$ i $\mathbf{c h} \mathbf{t}$ wie einige behaupten aus dem Unterscheidungsvermögen, sondern aus der Imagination.»

\title{
Die Befreiung der Tollhausnarren im Zeitalter der französischen Revolution ${ }^{1}$
}

Von Hans Karcher (Basel)

Die Befreiung der Tollhausnarren durch Pinel ist im Verlaufe der vergangenen hundert Jahre zu wiederholten Malen von Medizinern, Historikern und Staatsmännern in Nekrologen, Gedenkreden und gut dokumentierten Biographien gefeiert worden. Jedermann kennt daraus min-

1 Akademischer Vortrag, gehalten 12. Nov. 1946. 
destens eine der vielen Anekdoten über Pinel und sein Unternehmen. Aber nur wenige kennen das Hauptdokument derselben, nämlich das in seiner Schlichtheit gewaltige "traité médicophilosophique sur l'aliénation mentale ou la manie», in dem Pinel in übersprudelnder scheinbarer Unordnung sein Lebenswerk dartut. Es stellt ein Bändchen in octavo dar mit 125 Kapiteln auf 318 Seiten. Der Leser gewinnt daraus einen Einblick nicht nur in die Dramatik, sondern auch in die Problematik der großen ärztlichen Tat von Pinel, wobei offenbar wird, daß das Problem weniger darin liegt, daß die Tat getan wurde, sondern darin, daß sie gelungen ist.

Pinel hat, wie alle großen Bahnbrecher, Vorläufer gehabt. Er weist in seinem traité vor allem auf die großen Ärzte der Antike hin und nennt sie seine Vorbilder. Das klassische Altertum hatte das Irresein als Krankheit behandelt. Das christliche Mittelalter stempelte die Irrsinnigen zu Teufels- und Dämonenbesessenen. Die Reformation vermochte diese Auffassung nicht zu korrigieren. Der durch den Zeitgeist des fünfzehnten Jahrhunderts übersteigerte Glauben an den leibhaftigen Satan trug dazu bei, daß das christliche Abendland fortfuhr die Geisteskranken als Besessene zu betrachten. So kam es, daß kirchliche Instanzen sie bis in die Zeit der Aufklärung als Hexen auf dem Scheiterhaufen verbrennen ließen, während die Organe der weltlichen Macht sie in dunkle Verließe steckten und an Ketten fesselten, sie von rohen Gesellen gemartert in ihrem Unrate vermodern ließen. Durch die unmenschliche Behandlung wurden die Tollhausnarren zu tobsüchtigen Schreckensgestalten.

Wohl waren schon im sechzehnten Jahrhundert Ärzte aufgetreten, und hatten sich dafür eingesetzt, daß die Hexen als Geisteskranke aufgefaßt und die Irren als Kranke betreut werden sollten. Einer der berühmtesten unter ihnen war der Leibarzt des Herzogs Wilhelm III. von Jühlich, Cleve-Berg, J o $h$ a $n$ n W e y e $r, 1515-1588$, der in einer in vielen Ausgaben erschienenen Schrift wider den Hexenwahn ankämpfte, Geistliche und Ärzte der Mitschuld an den unsagbaren Leiden der ohne Grund zur Folter und zum Tode Verurteilten bezichtigte. Der Geist des Humanismus hatte in ihm Zweifel erweckt am Besessenheitswahn seiner Zeitgenossen. Im letzten Drittel des sechzehnten Jahrhunderts trat in Basel der Professor Praxeos und Stadtarzt F e l i x P l a t e r (1536-1614) für eine humanere Haltung der Irrsinnigen ein. Er zog die halluzinierenden Kranken aus ihren dunklen Zellen ans Tageslicht, erlaubte sogar bei Rasenden die Fesseln vorsichtig zu lockern, sofern sie weder sich noch andern Schaden zufügen konnten. Weder Weyer noch Plater ver- 
moehten es die Irrenpflege ihrer Zeit dauernd zu beeinflussen. Das Erbteil des Mittelalters war noch allzufest in den Gewohnheiten verankert, die Indolenz gegenüber der Not der Geisteskranken übermächtig.

Im 18. Jahrhundert war ein neuer Anfall von Hexen- und Wunderglauben über Europa hereingebrochen. Besonders in Süddeutschland und Österreich trieben Hexen und Zauberer ihr Unwesen und verfielen der blutigen Justiz der constitutio criminalis carolina. Damals griff Maria Theresia ein und überwies gefolterte und in bester Form zum Tode verurteilte Hexen ihren Leibärzten v a $\mathbf{n} \mathrm{S}$ w i e $\mathbf{t}$ e $\mathbf{n}$ und $\mathrm{d}$ e $\mathrm{H}$ a e $\mathrm{n}$ zur ärztlichen Begutachtung zu. Die armen gemarterten Tollhausnarren profitierten nicht von dem im Zeitalter der Aufklärung allerseits erwachenden Sinn für humanitäre Verpflichtungen. Wohl gliederten Joseph II. und seine Berater den Narrenturm in den Neubau des Wiener allgemeinen Krankenhauses ein, hielten aber die Irren wie ehedem angekettet in ihren Zellen. Trotzdem fing es an, da und dort zu tagen. Man entdeckte die Notlage der Irrenpflege. So zeitigte immerhin das 18. Jahrhundert einige bemerkenswerte Unternehmungen.

An den italienischen Hochschulen wirkten immer noch überragende ärztliche Persönlichkeiten. Unter ihnen befürwortete der im übrigen als Anatom bekannte Antonio Maria Valsalva (1666-1723) und in seinem. Gefolge der Florentiner Spitalarzt Vi n c en z o Chi a rugi (1753-1820) die seelische Kurmethode für die Behandlung der Geisteskranken. Chiarugi schuf in Florenz eine für die damalige Zeit mustergültige Irrenanstalt. In Deutschland erregte eine im gleichen Sinne geschriebene Dissertatio eines Arztes aus Paddenburg, namens Cornelius A lbert Kloekhof (1753) allgemeines Aufsehen. Aber es geschah nichts faßbar. Fortschrittliches.

-. In England waren es die Quäker, die die Geisteskranken menschenwürdig unterbrachten und verpflegten. Aber die englische Psychiatrie war im großen und ganzen nicht weniger rückständig als diejenige des Kontinentes. Das geht aus der Begutachtung der Geistesstörung des Königs Georg III. hervor. Und doch lag der Umschwung auch in England offenkundig in der Luft. 1798 schrieb der Chefarzt des Westminsterhospitals, Alexander Crichton, "an inquiry into the nature and origine of menthal derangement", in der er echte psychologische Kriterien in die Beurteilung des Irreseins einführte, den Leidenschaften und dem Affekt eine hohe Bedeutung für die Störung des geistigen Gleichgewichtes beimaß und sie als die gewöhnliche Ursache der Geistes- 
krankheiten bezeichnete. Die Schweiz blieb von der Bewegung nicht unberührt. Ende der achtziger Jahre des 18. Jahrhunderts unternahm es der Genfer Spitalarzt Abrah am Jolly (1748-1812), die Anwendung von Ketten und anderen Zwangsmaßnahmen auf seiner Irrenabteilung aufzuheben. Jolly wurde so in der Stadt von J. J. Rousseau zum Vorläufer von Pinel.

Als Pinel sein Befreiungswerk an den Geisteskranken in Gang brachte, fußte er demnach auf beachtenswerten Vorläufern. Aber es hatte sich bisher mehr um Worte als um Taten gehandelt. Wertvolle Anläufe waren ohne durchschlagende Dauererfolge geblieben. Pinel kommt das unbestrittene Verdienst zu, die Befreiung der Tollhausnarren aus ihren Ketten und Kerkern, die manchen vorgeschwebt hatte, die andere zaghaft versucht hatten, mit einer unwiderstehlichen Autorität für alle Zeiten durchgesetzt zu haben. Er erfaßte die günstige Konjunktur, die sich aus den schicksalsschweren Ereignissen der französischen Revolution ergaben, da assemblée und commune, betört durch die Utopien von J. J. Rousseau ebenso hemmungslos ihre Opfer der Guillotine zuführten, wie sie humanitäre Extravaganzen tätigten. Es entspricht durchaus der Psychologie der wildesten Epoche des revolutionären Umbruches, daß die französische Revolution auch der Verwirklichung erhabener Ideale freie Bahn gab. Die Erklärung der Menschenrechte schuf eine Grundlage, auf der den schmachvoll gepeinigten Tollhausnarren ihre Menschenwürde zurückgegeben werden konnte. Pinel machte nun diese Konjunktur seinem Unternehmen der Befreiung der Irren nutzbar und errang ihm unter dem Einsatze seiner selbst den endgültigen Erfolg. Die saluberrima facultas von Paris hatte ihn bei seiner Bewerbung um den Titel eines médecin régent dreimal durchfallen lassen. Die Convention machte dieses Unrecht wieder gut und übertrug 1793 Pinel die Chefarztstelle am hospice von Bicêtre mit dem Auftrage der gründlichen Sanierung dieses Hospitales. Bicêtre war damals ein riesiges Pandämonium des Jamimers und der Verzweiflung. Auf der einen Seite wurden dort die Mörder und Räuber, die den Galeeren zugeführt werden sollten, besammelt, auf der anderen Seite hauste das Heer der Kriegsinvaliden und dazwischen waren die Irren in feuchten niedrigen, übelriechenden Zellen untergebracht, auf Stroh gelagert, angekettet oder gefesselt, von rohen Wärtern ärger als Sträflinge gemartert. Pinel war von der Überzeugung erfüllt, daß die Abschaffung der $Z_{\text {wangsmittel auf der Irrenabteilung als die erste }}$ großzügige Maßnahme zu der ihm aufgetragenen Sanierung von Bicêtre 
durchgeführt werden müsse. Dazu bedurfte er der Genehmigung des Pariser Stadtrates.

Im September 1793 trat nun Pinel mit seinem Gesuche vor die commune von Paris. Die anwesenden Stadtväter waren in ihrer Mehrheit revolutionäre Extremisten. Vom Geiste Marats beseelt, spielten sie sich als Initianten des régime du salut public und der terreur auf, waren ständig auf der Lauer nach suspects. Der conventionel Couthon, das rabiateste Mitglied des Triumvirates Robespierre, Saint Justes und Couthon, präsidierte. Dieser verdächtigte Pinel, er wolle mit seinem Gesuche Volksfeinde verbergen und schalt ihn einen Aristokraten. Es war offenbar Couthon nicht entgangen, daß Pinel dem Kreise von Philosophen und Gelehrten angehörte, die im Salon der Madame Helvetius in Auteuil verkehrten und unter denen Lavoisier, Condorcet, d'Alembert, Thouret, Savary und die Arztphilosophen Cabanis und Roussel im öffentlichen Leben an exponierter Stelle standen. Dabei war aber Pinel rückhaltlos Anhänger der fortschrittlichen Freiheitsideen der Revolution. Er hatte allerdings keiner Assemblée angehört, hatte aber seine Bürgerpflicht als officier municipal und als Nationalgardist erfüllt, war als solcher dienstlich Zeuge von der Enthauptung des Königs gewesen. Er war darum in jeder Beziehung unverdächtig. Wir wissen nicht, was schließlich die commune bewog, Pinel ihr Placet zu geben. Sie erteilte Couthon den Auftrag, die Sache an Ort und Stelle zu prüfen. Tags darauf spielte sich in Bicêtre die viel beschriebene Szene ab. Der an beiden untern Extremitäten gelähmte Couthon kommt in seinem Fahrstuhl in Bicêtre angefahren. Gestützt von den starken Armen kräftiger Männer macht er seinen Rundgang durch das Spital. Aus allen Zellen wird er von den Geisteskranken angefaucht, angeschrien, insultiert, bedroht. Davon beeindruckt sagt er zu Pinel: "Ah, ça, citoyen, est-ce que tu es fou toi-même de vouloir déchaîner de pareils animaux?» Darauf Pinel: «Citoyen, j'ai la conviction que ces aliénés ne sont si intraitables que parcequ'on les prive d'air et de liberté.» Worauf Couthon mit den Worten seine Zustimmung gab: «Eh bien, fais-en ce que tu voudras; mais je crains bien que tu ne sois victime de ta présomption.»

Der erste mit zwölf Irren angestellte Versuch gelang so gut, daß Pinel ermutigt wurde, sein Befreiungswerk im Großen durchzuführen. Dabei erfreute er sich der Beihilfe des vorzüglichen .Concierge von Bicètre mit Namen Pussin. Er nennt diesen «un des hommes les plus expérimentés 
dans l'art de diriger les aliénés et le plus propre par sa fermeté courageuse à maintenir un ordre invariable dans l'hospice» und rühmt seinen zèle, seine intelligence und seine principes d'humanité.

Trotz allem stellte sich die Befreiung der Irren zur Zeit, da sich die Revolution in den grausamsten Auswüchsen austobte, als ein recht ungewöhnliches Unternehmen heraus. Einige Beispiele unter vielen mögen das illustrieren.

Einmal drang ein bewaffneter Volkshaufe in das Hospice von Bicêtre ein, in der Absicht Opfer der alten Tyrannei, die unter den Irrsinnigen verborgen sein mochten, zu befreien. Mehr oder weniger betrunken, wie diese Leute waren, zogen sie von Zelle zu Zelle, alle Insassen ausforschend. Überall war der Irrsinn offenkundig. Da, auf einmal stießen sie auf einen scheinbar stillen Mann, der sich beklagte, widerrechtlich gefangen gehalten zu werden und der sie anflehte, ihn zu befreien. Mit wilden Imprekationen und tätlicher Bedrohung des Concierge befreiten sie ihn und schleppten ihn im Triumphe, unter dem Rufe: "Vive la République» fort. Doch kaum ins Freie gelangt, ergriff der vom Tumulte und vom Gebrüll seiner Befreier in Erregung versetzte Irre den Säbel des einen von ihnen, fuchtelte damit in der Luft herum, sodaß links und rechts Blut floß. Seine Befreier hatten Mühe ihn zu bändigen, zu fesseln und ihn in seine Zelle zurückzubringen. Wutschnaubend zogen sie von dannen.

Ein anderes Mal geriet Pinel selber in einem Volksauflaufe in höchste Lebensgefahr. Von einer fanatisierten Horde als aristocrate mit dem Aufhängen an der Laterne bedroht, wurde er nur durch das kraftvolle Eingreifen seines getreuen Dieners Chevingé gerettet. Dieser Chevingé war von Pinel als einer der ersten aus den Ketten einer Irrenzelle befreit worden. Er war ein ehemaliger Soldat und erwies seinem Befreier seinen Dank durch einen ebenso kunstgerecht wie unaufhaltbar durchgeführten Gegenangriff auf seine Bedroher.

Ein weiteres Mal - es war im Jahre III der Republik - glaubten die Verwalter der Zivilspitäler in einer Anwandlung revolutionären Übereifers die Kreuze, Heiligenbilder und Marienstatuen aus den Krankensälen und aus den Kapellen der Spitäler entfernen zu müssen. Es war schon abends spät geworden, als sie in Bicêtre eintrafen, sodaß sie bloß einen Teil ihres Zerstörungswerkes durchführen konnten. Sie versprachen wiederzukommen. Für Pinel war dies eine widerliche Situation. Er war darüber beunruhigt, wie seine Kranken darauf reagieren würden, wenn ihre Heiligtümer entheiligt würden. Ein Ausweichen war nicht 
möglich. Nach einem Kriegsrate mit seinem Concierge wurde beschlossen, den Zerstörern zuvorzukommen. So überließ es Pinel dem Pussin die Irren selbst die kirchlichen Embleme in einer Art patriotischer Extase auf dem Altare der Nation opfern zu lassen. Pussin erfüllte den Auftrag in geschickter Weise, allerdings nicht ohne daß die Kirchenschändung die depressiven und melancholischen Kranken in schwere Seelennot gebracht hätte.

All dies konnte geschehen, weil nach althergebrachter Sitte der Zutritt zu den Tollhäusern den Besuchern freistand.

Neben diesen dramatischen Intermezzi gab es im täglichen Spitalbetrieb unerhörte Schwierigkeiten, die durch die prekären Zustände des Hauses sowie durch die personellen Verhältnisse bedingt waren. Besonders schlimm stand es mit der Spitalverwaltung, die im Sturm und Drang der Revolution einem steten Wechsel ausgesetzt war und die in ihrer Bedrängnis und Indolenz dem Chefarzte mannigfaches Ungemach bereitete. Pinel meisterte alle diese Mühsale, unter selbstloser Hingebung, mit unbeirrbarer Zuversicht. In seiner Persönlichkeit liegt demnach der Kern des Problemes der Befreiung der Irren. Wir müssen hier einige biographische Daten einschalten.

Philippe Pin el 1745-1826 entstammte einer Ärztefamilie aus dem Languedoc. Sein Vater ließ ihn im Seminar der Pères de la Doctrine ausbilden. Die Pères doctrinaires bekannten sich zur Ordenslehre der Congrégation de l'oratoire de Jésus. Wie diese, erzogen sie ihre Zöglinge zur reinsten Form der christlichen Doktrin. Außerdem vermittelten sie ihnen solide humanistische und philosophische Kenntnisse. Aufgemuntert durch den Bischof von Lavaur wurde Pinel Priester und brachte es bis zu den «ordres mineurs». Aber es fehlte ihm an der innersten Berufung zum Priestertum. Die Mystik der Patres widerstrebte seiner ureigensten Sinnesart. So kam es, daß er sich den Lockungen des Zeitgeistes hingab, sich für Voltaire und J. J. Rousseau begeisterte. Mit 22 Jahren verließ er die Pères doctrinaires, zog den Priesterrock aus und ließ sich in Toulouse als freier Student immatrikulieren. In Toulouse widmete er sich den exakten Wissenschaften, trieb eifrig Mathematik, wurde magister artium und 1773 Doktor der Medizin. Dann folgten vier Jahre intensiven Studiums in Montpellier, während welcher Pinel seine Kenntnisse in allen Fächern der Medizin vertiefte, die Schriften der medizinischen Autoren der Antike im Urtexte las und die zeitgenössische Literatur in der jeweiligen Landessprache zu studieren sich bemühte. Er 
lernte damals englisch bei einem englischen Kommilitonen. Sein Lieblingsautor war der 1707 verstorbene römische Kliniker G i o r g i o B a g livi, dem er seine Abneigung gegen alle Systeme und Hypothesen entnahm. So kam es, daß er sich in Montpellier von der dorthin aus Halle a. S. importierten animistischen Doktrin von $\mathrm{G}$ e or $\mathrm{g} \mathrm{S} t$ a $\mathrm{h} l$ fern hielt. Als dann $B$ a $r$ t h è s aus ihr seine vitalistische Lehre herausentwickelte und in metaphysischen Diskussionen das Prinzip des Lebens zu erklären versuchte, da widersprach dies seiner naturwissenschaftlich eingestellten Sinnesart. Pinel fuhr fort, Mathematik zu treiben und veröffentlichte einige zoologische Arbeiten, die beachtet wurden. Die angusta res domi zwang ihn hier, wie einst in Toulouse, seinen Unterhalt mit Privatunterricht $\mathrm{zu}$ bestreiten, was ihm allerdings interessante Freundschaften einbrachte. Der um elf Jahre jüngere Chemiker J e a n A n t o in e C h a p t a l, der unter dem Konsulate von Bonaparte Innenminister werden sollte, blieb sein Leben lang mit Pinel freundschaftlich verbunden. Ihre beidseitige Verehrung für Rousseau hatte sie einander nahe gebracht.

Pinel war mit 29 Jahren nach Montpellier gekommen; war auch dort zum Doktor kreiert worden. Mit 33 Jahren wanderte er, wie einst die Scholaren, zu Fuß nach Paris. Es war im Jahre 1778, dem Todesjahr von Voltaire und J. J. Rousseau. Sein erster Besuch galt Chaptal. Die beiden Freunde pilgerten zum Grabe von Rousseau in Ermenonville. Es widerspricht aber eben so sehr den Tatsachen als auch der Wesensart von Pinel, wenn erzählt wird, er habe damals aus lauter Verehrung für den großen Philosophen fünf Tage und Nächte schlaflos verbracht und sich dabei kaum Zeit zum Essen gegönnt. Die Ernüchterung gegenüber J. J.Rousseau ließ übrigens nicht lange auf sich warten. Beide Freunde fühlten sich angewidert durch die Schamlosigkeiten seiner «Confessions» und durch die pathologisch anmutenden Verfolgungsideen der Rêveries du promeneur solitaire».

Pinel fand sich in Paris bald zurecht, darbte sich mit seinen kümmerlichen Ersparnissen durch die ersten Monate hindurch, zielbewußt und mit klarem Verstande die Bildungsmöglichkeiten der Hauptstadt ausnützend. Einflußreiche Gelehrte, unter ihnen d'Alembert, verhalfen ihm zu einträglichem Privatunterricht. Dann trieb er wissenschaftliche Journalistik, schrieb Artikel in das Journal de physique und wurde 1784 Leiter der Gazette de santé. In jenen Jahren übersetzte er die Institutionen von Willi a m C ullen ins Französische und besorgte eine Neuauflage der Praxis medica von B a livi. 
Der Freundeskreis, der sich im Salon der Madame Helvetius traf, huldigte der sensualistischen Philosophie von J o h $\mathrm{n} \mathrm{Locke} \mathrm{und} \mathrm{seines}$ französischen Nachbeters Co ndilla c. Locke vertrat bekanntlich in seinem «Essay concerning human understanding» die Auffassung, daß die Sinnesempfindung und die aus ihr hervorgehende Sinneswahrnehmung die einzige Quelle für die Erkenntnis der äußeren Gegenstände ist. Locke lehnte jede angeborene Idee oder Erkenntnis ab. Er forderte von der Philosophie, daß sie sich einzig und allein auf die Methode der Beobachtung, der Induktion und des Experimentes stütze und darauf verzichte, sich transzendentalen Problemstellungen über die Substanz, das Wesen und die intime Konstitution der Dinge hinzugeben. Diese jeder Spekulation abholde Philosophie machte Locke zum Lieblingsphilosophen der Ärzte des 17. und 18. Jahrhunderts. T h o m a s S y d e n h a m war mit Locke eng befreundet gewesen. Pinel kannte ihn vom Seminar der Pères doctrinaires her. Der Abbé E t i e n n e B on not de Condilla c übertrug die Anschauungen von Locke ins Französische und fügte ihnen formvollendet komponierte Ausweitungen an, über die Äußerungen des Geistes durch Zeichen, Gesten, Laute, Sprache, Gesang usw.

Als Pinel Psychiater geworden war, erschlossen ihm die Ausführungen Lockes die Erkenntnis des krankhaften Geschehens in der Psyche der Geisteskranken. Er folgte Locke in seiner Analyse der Geistesoperationen, in seinen Untersuchungen über das Entstehen der einfachen Ideen, über die Gestaltung der Relationen aus dem Vergleiche zweier Dinge, über die Funktionen der Perzeption und des Gedächtnisses, über den Willen und die Freiheit, über die Modifikationen der Geistesarbeit, wie sie durch Lust- und Unlustgefühle, durch Freude und Schmerz oder durch Leidenschaften hervorgerufen werden.

Die Biographen von Pinel wollen wissen, daß er, durch den Selbstmord eines Geistesgestörten Freundes beeindruckt, der Psychiatrie zugeführt wurde. 1783 trat er in den ärztlichen Stab der Privatirrenanstalt des Dr. Belhomme ein. Da bekam er die Unzulänglichkeit der damaligen psychiatrischen Kenntnisse und die Trostlosigkeit der irrenärztlichen Kurmethoden vordemonstriert. Vergebens fahndete er nach verwendbaren Ratschlägen in den Spitälern der Hauptstadt oder im damaligen Schrifttum. Überall stieß er auf unverantwortliche Routine. So entschloß er sich zu den Grundsätzen der Ärzte der Antike zurückzukehren. Während der fünf Jahre seiner Tätigkeit an der Klinik Belhomme sammelte er 
als Autodidakt wertvolle Beobachtungen, die ihm den Stoff zu mehreren Publikationen lieferten. Bei der Lösung einer 1791 von der Société de médecine gestellten Preisaufgabe über die Mittel der Behandlung der noch nicht dem Greisenalter verfallenen Geisteskranken, erklärte er sich als unbedingter Anhänger der psychischen Kurmethode.

Am 25. August 1793 wurde Pinel Chefarzt von Bicêtre, das er am 13. Mai 1795 verließ, um die ärztliche Leitung der Salpétrière zu übernehmen. Bicêtre war ein Männerhospital, die Salpétrière beherbergte ausschließlich Frauen. 1794 erhielt er den Lehrstuhl für physique médicale und Hygiene und 1795 den Lehrstuhl für innere Pathologie an der neugegründeten école de santé. Damit wurde Pinel klinischer Lehrer und Chef der nach ihm benannten Schule der Salpétrière. Als solcher faßte er zu Handen seiner Schüler seine Vorlesungen in einem Lehrbuche: "Nosographie philosophique» (1798) zusammen. Wir müssen darauf verzichten, auf dasselbe einzugehen. Es würde uns allzuweit von unserem heutigen Thema ablenken. Seine Schüler schätzten an diesem Lehrbuche die klare Formulierung des Stoffes in prägnanten, leichtfaßbaren Lehrsätzen. Sie fühlten sich vor allem angezogen durch das gütige Verständnis, das ihr Lehrer jedem von ihnen entgegenbrachte.

Dem klinischen Scharfsinn von Pinel ist es zu verdanken, daß sein Befreiungswerk an den Irren gleich beim ersten Versuch Erfolg hatte. Es gelang ihm aus dem wilden Gewirr der Narren von Bicètre jene zwölf Fälle herauszufinden, die geeignet waren, das Experiment gelingen zu lassen. Als er in der Folge daran ging, den zweihundert übrigen Insassen von Bicêtre eine menschenwürdige Haltung und freie Bewegung innerhalb der Anstalt zu geben, bedurfte es einer hohen klinischen Einsicht, um dieselben so zu gruppieren, daß ihre Befreiung aus den Ketten und aus dem Unrat ihrer dunkeln Behausungen reibungslos geschehen konnte. Dabei war der bewunderte klinische Blick von Pinel keine ihm im Schlafe geschenkte Gottesgabe, sondern das Ergebnis einer mühevoll erarbeiteten klinischen Erfahrung. Pinel verweilte Stunden und Aberstunden bei seinen Kranken, führte Krankengeschichten, nahm sorgfältige Anamnesen auf und bemühte sich, den Irrsinn jedes einzelnen seiner Kranken nach den Regeln exakten naturwissenschaftlichen Forschens zu ergründen. Trotzdem sich im traité médicophilosophique de l'aliénation mentale von Pinel unzählige Anläufe zu psychiatrischen Erkenntnissen finden, zu denen die spätere Forschung des 19. Jahrhunderts gelangen sollte, so war es ihm nicht gegeben, über eine einfache Klassifikation der Psychosen hinaus 
zu gehen, die mehr oder weniger schon den Ärzten des Altertums geläufig war. Pinel schied seine Kranken von Bicêtre in fünf empirisch aufgestellte Kategorien: 1. die einfache oder mit Hypochondrie komplizierte Melancholie, 2. die manische Tobsucht ohne Delirien, 3. die Manie mit Delirien und mit Verwirrung des Geistes, 4. die Demenz, 5. die Idiotie.

Seine Sendung lag weniger in der wissenschaftlichen Ergründung des Irreseins als in der Behebung des grausamen Schlendrians der traditionellen Behandlung der Tobsucht. Diese bestand in massiven, bis zur Erschöpfung des Patienten getriebenen Aderlässen, Douchen, Untertauchen im kalten Bade. Sie war durch B o erh a a ve in seinen Aphorismen empfohlen worden und wurde im Pariser Hotel Dieu den erregten Irren zugefügt. Pinel geriet jedesmal in Zorn, wenn ein manischer Patient, dessen Erregung durch diese Behandlungsweise ins Unermeßliche gesteigert worden war, gefesselt in das Hospice von Bicêtre eingeliefert wurde. Er fühlte sich durch derartige Vorkommnisse aufgestachelt, seine psychische Kurmethode, sein «traitement moral» bei den erregten Geisteskranken schon bei ihrem Eintritte in die Irrenanstalt anzuwenden. Dabei mahnte er eindrücklich, man möge sich der Maximen der Alten, vor allem des Celsus und des Coelius Aurelianus erinnern. Aber es genüge nicht, daß man es bei den allgemeinen Leitmotiven bewenden lasse, man müsse sich die Kunst aneignen, die Irren zu trösten, sich mit Wohlwollen mit ihnen zu unterhalten, ihnen gegebenen Falles ausweichende Antworten zu geben, um sie nicht durch die Ablehnung eines Wunsches zu verbittern. Es gebe Fälle, denen man eine wohltuende Furcht einjagen müsse, um ihren unbeugsamen Starrsinn ohne Gewaltanwendung zu meistern. Nur derjenige werde Erfolg haben, der im strengsten Sinne des Wortes zu individualisieren verstehe. Unerläßlich sei dabei die Selbstkritik, die die Mißerfolge ebenso wahrheitsgetreu wiedergebe wie die Erfolge, unerläßlich sei aber auch der Glaube an die Heilbarkeit mancher Formen des Irreseins, damals eine revolutionierende Ansicht. Demnach weist die Abschaffung der Zwangsmittel in der Irrenpflege durch Pinel neben der negativen, abbauenden, eine positive aufbauende Seite auf, wobei letztere die erstere beträchtlich überragt.

Dabei war Pinel bemüht, mit seinem traitement moral causale, ätiologische Therapie zu treiben. Nach dem Vorgehen von Locke und Condillac suchte er die Störungen der Geistesfunktionen, die zum Ausbruch des Irreseins geführt hatten und die Anlaß zu Erregungszuständen gaben, 
zu erfassen. Er fand sie vor allem in einer Störung des Willens in denjenigen Fällen, bei denen das Erinnerungsvermögen, das Urteilsvermögen und die Phantasie erhalten waren. Der durch den Willen nicht bemeisterte Affekt fesselte seine Aufmerksamkeit und führte ihn zwangsläufig zu den diesen Affekt auslösenden Leidenschaften. Auf der Männerabteilung von Bicêtre überragte zur Zeit der Revolution die Gier nach Ruhm, nach politischem, militärischem Ruhm, der Wunsch wegen einer Großtat in die Geschichte einzugehen alle übrigen Leidenschaften. Reminiszenzen aus der Antike verliehen ihnen heroisch klingende Ausdrucksformen. Im Irrsinn ergingen sich der zeitbedingte Machthunger, die Haßgedanken, die Rachsucht oder der Schrecken in ungezügelter Rede. Von Liebe ist im traité von Pinel kaum die Rede. Das sexuelle Moment ist nicht erwähnt. Für ihn resümierte sich das Problem der Liebe in einer glücklichen Ehe mit einer Frau, die seiner Sendung vollstes Verständnis entgegenbrachte und die ihm wohlgeratene Söhne schenkte.

Während Pinel mit seinen manischen Kranken im ganzen genommen gut fertig wurde, so gestaltete sich das traitement moral bei den melancholisch Deprimierten umso schwieriger, besonders wenn sie von religiöser Not gequält waren. Es möchte scheinen, als hätte Pinel die Caritas, die Kunst zu hören, zu begreifen und zu verzeihen aus der Zeit, als er bei den Pères de la doctrine die ordres mineurs erhalten hatte in das System seiner psychischen Kurmethode hinüber gerettet. Dabei bemühte er sich, sein traitement moral frei von jeder geistlichen Mystik verstandesmäßig nach nüchternen ärztlichen Grundsätzen aufzubauen. Während der entscheidenden Jahre war seine Weltanschauung eher philosophisch als christlich eingestellt. In seinem traité erzählt er, wie er einen Kranken von den ihn quälenden religiösen $Z$ wangsideen dadurch befreite, daß er ihm Spottverse auf die Religion von Voltaire vorlas. Anderseits steht fest, daß er sich während der schlimmsten Wochen der Terreur kirchlich trauen ließ. Das sind wohl mehr zeitgebundene scheinbare Widersprüche als der wirkliche Ausdruck seines ureigensten Wesens. Dieses ist kaum von jemandem prägnanter erfaßt worden als von Edouard Herriot, als er in seiner Ministerialrede an der Jahrhundertfeier für Pinel sagte: «Nulle figure ne fut plus attrayante, plus rayonnante que celle du ,bon Pinel' ... Elle commande l'hommage le plus sincère, elle prouve une fois de plus, la force de l'intelligence, mais elle démontre aussi, tout ce qu'il peut y avoir de génie dans la bonté».

Pinel empfand weniger Freude an den Heilerfolgen, als Sorge um das 
Versagen seines traitement moral bei den Tobsüchtigen, bei denen er trotz liebevollster Betreuung immer wieder zur Fesselung greifen mußte, wenn ihre Ausbrüche sich und andern gefährlich wurden. Entsprechend seiner Auffassung der Tobsucht als einem Versagen des Willens, betrachtete er die Fesselung als eine Züchtigung, als ein erzieherisches Mittel, das die Kranken von der Notwendigkeit sich zu beherrschen, überzeugen sollte. Er ließ darum die Fesselung in der Zwangsjacke, verbunden mit der Isolierung in der Tobzelle nur wenige Stunden dauern und hob sie auf, sobald er fand, daß der Patient einer freundlichen Zurede wieder zugänglich geworden war.

Das traité von Pinel erzählt von dramatischen Szenen, die sich dabei abspielten. Man muß sich bloß wundern, wie glimpflich sie ausgingen, wenn man bedenkt, welch heikle Aufgabe dem Chefarzt von Bicêtre daraus erwuchs, daß er aus verwilderten Tollhausnarren gesittete Geisteskranke und aus rohen, dem Schlendrian ergebenen Tollhauswärtern hingebende Irrenpfleger machen mußte, und das zu einer Zeit, da sich ein in Raserei verfallener Pöbel vor den Toren des Spitals den tollwütigsten Exzessen hemmungslos hingab. Zusammen mit seinem Oberwärter Pussin brachte es Pinel zustande, in den zwei Jahren, da er Bicêtre vorstand, ein gut geschultes, zuverlässiges, jederzeit einsatzbereites Pflegepersonal zu gewinnen.

Folgendes Beispiel demonstriert Disziplin und Können des Personals und gleichzeitig die von Pinel angewandte Taktik der Übermacht gegenüber gefährlich erregten Patienten. Ein junger Patient, der sich seit mehreren Monaten ruhig, frei im Spital bewegt hatte, geriet plötzlich in einen Anfall von Raserei, schlich sich in die Küche und erwischte dort ein Hackmesser, das zu Verhacken des Gemüses diente. Als ihn das Küchenpersonal gewaltsam entwaffnen wollte, steigerte sich seine Wut. Er sprang auf den Küchentisch und bedrohte jeden, der ihm nahe kommen wollte mit dem Kopfabschneiden. Da kam die leitende Schwester dazu, gab sich, als verurteile sie seine Verfolger: "Warum hindert ihr diesen starken Mann mit mir zusammen zu arbeiten?», sprach zum Rasenden freundlich und rief ihn furchtlos zu sich, zeigte ihm, wie er sich seines Hackmessers bedienen könne, um die Gemüse zu verhacken, lobte ihn als einen wertvollen Gehilfen bei ihrer Arbeit. Dadurch getäuscht, versenkte dieser sich in seine Arbeit und konnte auf ein gegebenes Zeichen hin vom Pflegepersonal umringt, überwältigt und in seine Zelle 
verbracht werden, ohne daß irgend jemand etwas zu Leide geschehen wäre.

Pinel war der Ansicht, daß das körperliche Wohlbefinden und das körperliche Wohlgefühl seinen Kranken die Grundbedingung für das Gelingen des traitement moral sei. Die Hebung der Körperpflege, des Komforts - sit venia verbi - in den Wohnzellen und Aufenthaltsräumen, der Hygiene der Wasch- und Badegelegenheiten, sowie vor allem der Latrinen, der Maßnahmen für die Entfernung der Abfallstoffe, die sorgfältige Bereitung und leckere Darreichung der Nahrungsmittel waren seine stete Sorge. Er stellte sich die Aufgabe, das mittelalterliche Tollhaus von Bicêtre in eine Heil- und Pflegeanstalt umzugestalten. In Praxi handelte es sich vorläufig um ein Ausmisten, Fegen, Bürsten, Reinemachen, Lüften. Es fehlte an der genügenden Zufuhr von Wasser, an der Heizung, an den genügenden Krediten für die dringendsten Bedürfnisse.

Entsprechend seiner zuversichtlichen Einstellung hinsichtlich der Heilung galt sein Interesse vor allem den einer Heilung zugänglichen Kranken und den Rekonvaleszenten, wie er sie nannte. Um sie vor der Rückfallsmöglichkeit, die sich aus dem Zusammenleben mit Schwerkranken ergeben konnte, zu schützen, gruppierte er die Anstaltsinsassen nach der Schwere ihres Leidens, schuf eigentliche Rekonvaleszentenabteilungen, in denen eine nach der Begabung, dem beruflichen Können und den Körperkräften individualisierende Arbeitstherapie in der Behandlung eine zentrale Bedeutung erhielt.

In Bicêtre konnte es sich vorerst bloß um Ansätze zu alledem handeln. Erst als Pinel an die Salpétrière berufen wurde, vermochte er es, seine Pläne einigermaßen zu verwirklichen. Die Lokalitäten der Salpétrière boten bessere Möglichkeiten zur Sortierung der Krankheitsfälle und zur Arbeitstherapie. Trotzdem war die Aufgabe, die ihm hier zufiel, eine gewaltige. Da galt es 600 irrsinnige Frauen zu meistern und die wilde Meute z. T. recht bedenklicher Wärterinnen der neuen Methode der Irrenpflege dienstbar zu machen.

Pinel fiel zur Zeit der Restauration einer Säuberungsaktion der Ultras zum Opfer. Er mußte sein Amt quittieren. 1822 erlöste den 80jährigen ein Schlaganfall von der Trübsal dieser Welt. Er hatte das Glück gehabt, daß eine Equipe hochbegabter, gleichgesinnter junger Psychiater aus seiner Schule hervorgingen. Einzelne unter ihnen kamen schon zu seinen Lebzeiten in leitende Stellungen in Bicêtre und an der Salpétrière. F e $\mathrm{r}$ rus, Falret, Voisin, Georget haben das Erbe von Pinel in 
Ehren verwaltet. Sie alle überragte aber E s q u i r o l, erst leitender Arzt einer Abteilung der Salpétrière, später Chefarzt der Königlichen Irrenanstalt von Charenton. J e a - E t i en n e-Dominique Es quirol (1772-1840) stammte wie Pinel aus dem Languedoc, war wie er Priester gewesen, bevor er Mediziner wurde. Auch in bezug auf ärztliche Ethik, medizinischen Forscherwillen, philanthropische Hilfsbereitschaft, gütiger Bescheidenheit und Zähigkeit im Durchsetzen seiner Pläne war er Pinel nicht unähnlich. Aber er überragte ihn um vieles an Organisationstalent.

Als diese jüngere Generation ans Werk ging, stand es schlimm um die Irrenabteilungen. Mit dem Ausklang des napoleonischen Epos waren allerlei Kriegsseuchen über Frankreich hereingebrochen, Unterleibstyphus, Flecktyphus, Ruhr, Tuberkulose wüteten katastrophal in Heer und Volk. Alle Spitäler und mit ihnen alle Irrenabteilungen waren mit den Kriegsopfern über die Maßen belegt worden. 1818 inspizierte Esquirol auftragsgemäß die Irrenanstalten von Frankreich. Er publizierte seinen Bericht an das Ministerium des Innern in einer Schrift. Als die öffentliche Meinung davon Kenntnis bekam, wallte sie entrüstet auf, so daß die Indolenz der Regierung aufgerüttelt wurde. Esquirol schrieb damals: "Une maison d'aliénés est un instrument de guérison, entre les mains d'un médecin habile, c'est l'agent thérapeutique le plus puissant contre les maladies mentales.»

Auf diesem fundamentalen Prinzip baute er einen generellen, peinlichst durchgearbeiteten Plan für den Bau und den Betrieb, sowie für die Organisation des Personales einer Irrenanstalt auf, der es ihm ermöglichte, das Tollhaus von Charenton in eine Heil- und Pflegeanstalt für Geisteskranke umzuwandeln. Er verwirklichte da alle Postulate von Pinel hinsichtlich der Lage auf dem Lande, der reichlichen Wasserzufuhr, der hygienischen Einrichtungen der Wohn- und Aufenthaltsräume der Kranken, der Isolierzimmer, der Arbeitsräume, Hebung des Niveaus des Pflegepersonals durch angemessene Löhnung und Sozialversicherung usw. Er setzte es durch, daß dem Chefarzt die oberste Leitung der Anstalt zuerkannt wurde. Der administrative Direktor wurde ihm untergeordnet. Er wurde der Spiritus rector der Haltung des ganzen Hospitales. Nach seinem Vorschlage wurde eine Aufsichtskommission bestellt. Diese war Wahlbehörde für die Funktionäre der Anstalt. Der Chefarzt legte ihr jedes Jahr einen Rechenschaftsbericht über den Gang der Anstalt vor. Esquirol hat in einem «mémoire historique et statistique sur la maison de Charenton 1835» die Entstehungsgeschichte und die Entwicklung von 
Charenton geschildert und an Hand von einwandfreien Statistiken und autentischen Aufzeichnungen seinen eigenen Beitrag zu dem fortschrittlichen Gedeihen des größten Irrenspitals jener Zeit dargetan. Esquairiol erweist sich da als der unbestrittene Bahnbrecher auf dem Gebiete der Anstaltspflege der Geisteskranken. Die wissenschaftliche Psychiatrie verdankt Esquirol eine Reihe neuer Erkenntnisse, unter denen ich bloß die wichtigsten nenne: Die Ausschälung der Monomanie aus dem Sammelbegriff der manischen Zustände, die scharfe Umschreibung der dementia paralytica, wobei seine Schüler Ca l m e il und B a y l e das Krankheitsbild der progressiven Paralyse und deren anatomisches Substrat überzeugend darstellten.

In England war die von Pinel eingelegte Saat auf einen gut vorbereiteten Boden gefallen. Im ersten Drittel des 19. Jahrhunderts hatten begüterte Engländer, dem Gefühle der sozialen Verpflichtung und der Gemeinnützigkeit folgend, vielleicht auch bewegt durch das ihnen eigene nationale Selbstgefühl, die Irrenanstalten mit reichlichen Zuschüssen dotiert, die es denselben gestatteten, die Irrenpflege in jeder Beziehung zu heben. So wurde die Voraussetzung geschaffen, auf der J o h n C o n nol y (1796-1866) sein «non restraint System» aufbauen konnte. Connoly war 1839 Hausarzt am Middleessex-Asyl geworden und hatte dort mit seinem non restraint System die letzten Überreste der mechanischen Zwangsmittel ausgeschaltet. Er suchte die Beruhigung seiner erregten Patienten einzig und allein mit Geduld, Güte, durch hochwertige Wartung, Komfort, Reinlichkeit, gute Kleidung zu erwirken. Ein gewisses Zeremoniell bei den Mahlzeiten, leckere Darbietung der Speisen, sollte dazu beitragen, den Kranken Haltung zu verleihen. Als Conolly sein System veröffentlichte, erhob sich eine Kontroverse zwischen den Pariser Psychiatern und Conolly hinsichtlich der Behandlung der akuten, Gefahren bedingenden Tobsuchtsanfälle der manischen, epileptischen und paralytischen Irren. Die Franzosen pflegten sie mittelst der Anlegung der Zwangsjacke unschädlich zu machen, währenddem sie Conolly in gut gepolsterten Isolierzimmern in der «Seklusion», wie er das nannte, sich austoben ließ, nachdem er sie durch handfeste Wärter hatte überwältigen lassen. Der Streit drehte sich darum, was barbarischer sei, die Zwangsjacke oder die Wärterfäuste, während doch der springende Punkt der Kontroverse darin liegt, daß die Pariser Ferrus, Falzet und Voisin, dem Beispiele von Pinel folgend, die Fesselung als eine Art Strafmaßnahme für ungebührliches Benehmen ansahen, während für Conolly die Seklusion 
bloß ein Mittel zur Beruhigung sowie zum Schutze der Umgebung und der Kranken selbst darstellte.

In Deutschland machte die Reform der Irrenpflege im ersten Drittel des 19. Jahrhunderts einen eigenartigen Entwicklungsgang durch. Die Kunde von der Befreiungstat von Pinel drang nach Wien und Berlin. In Wien besorgte M. W a g n e r 1801 eine deutsche Übersetzung des traité von Pinel unter dem Titel: «Abhandlung über die Geistesstörungen». In Berlin schrieb der Verfasser der jedem Mediziner bekannten Schrift über die Lebenskraft, J o h. C h r i s t i a n R e i l 1759-1813, "Rapsodien über die Anwendung der psychischen Kurmethode auf Geisteszerrüttungen» 1803, ein ideenreiches, temperamentvoll geschriebenes Buch. Reil gilt als der Verbreiter der Kurmethode von Pinel in Deutschland. Dabei schreibt er unartig geringschätzig über ihn. Reil vermochte keinen nachhaltigen Einfluß auszuüben, weil er einerseits über keine praktische Erfahrung verfügte und weil er anderseits ganz im Banne der Naturphilosophie Schellings war. Die deutsche Medizin wurde bekantlich im Zeitalter der Romantik durch die Philosophie von Schelling mit ihrem wortreichen, substanzlosen, jeden Fortschritt hemmenden Geschwätz in einen betrüblichen Irrgang hineingedrängt. W u n d e r l i c h, der diese Epoche der deutschen Medizin als Student erlebt hatte, stellt sie in seinen Vorlesungen über die Geschichte der Medizin als recht düster dar. Dem gegenüber weiß K r a e p e l i $n$ in seiner Schrift «Hundert Jahre Psychiatrie» auch dieser Zeitperiode allerlei Wertvolles zu entnehmen. Tatsächlich bemühten sich da und dort die leitenden Ärzte von Landirrenanstalten, die Haltung ihrer Kranken menschenwürdig zu gestalten. In Berlin scheiterten alle fortschrittlichen Bemühungen am Starrsinn der Traditionalisten unter den Ärzten. So konnte es möglich werden, daß noch 1818 ein Oberarzt des Charitékrankenhauses, namens H o r n, über neue von ihm erfundene oder verbesserte $Z_{\text {wangsmethoden }}$ und Maschinen, über eine Drehmaschine und einen Drehstuhl berichten und dabei sich einbilden konnte, in vielen Fällen von Geisteskrankheit höchst wohltätige Wirkungen erzielt zu haben.

Der entscheidende Umschwung trat in Deutschland erst ein, als W i l helm Griesinger (1817-1868) bei Conolly das non restraint System kennen lernte und dasselbe erst in Zürich und 1860 an der Irrenabteilung der Berliner Charité systematisch durchführte. Aber die endgültige Lösung des Problems kam erst, als die Deutschen Irrenärzte, unter der Führung von $\mathrm{K}$ r a e p eli n, den Wachsaal einführten. Im Kraepe- 
lin'schen Wachsaale stehen die erregten Geisteskranken unter ständiger Bewachung, werden da mittelst planmäßig durchgeführter Bettbehandlung und richtig dosierter Schlafmittel beruhigt. Der Wachsaal von Kraepelin bildet gewissermaßen die letzte Erfüllung der durch Philipp Pinel im Zeitalter der Französischen Revolution in Gang gebrachten Befreiung der Tollhausnarren aus ihren Ketten und Kerkern, der menschenwürdigen Pflege und Wartung der Geisteskranken. Die Tollhausnarren sind heute nur noch ein historischer Begriff. Dem Ingenium, dem medizinischen Wissen und ärztlichen Können, sowie der selbstlosen Hingabe ethisch hochstehender Irrenärzte, von Pinel über Esquirol, Conolly bis zu Kraepelin, kommt das Verdienst zu, die Schande der abendländischen Christenheit getilgt zu haben, während Jahrhunderten ihre Geisteskranken als Tollhausnarren eingekerkert, gefesselt und gemartert zu haben.

Schrifttum : L. Madelin : La Révolution. 1912. Mignet : Histoire de la révolution française. $\mathrm{Kir} \mathrm{ch}$ h of $\mathrm{f}$, Theod.: Geschichte der Psychiatrie und Grundriß der Geschichte der deutschen Irrenpflege. G o ld h a h n, Richard: Spital und Arzt von einst und jetzt. 1940. L o c k e, John: Essay concerning human understanding. C o ndill a c, Etienne: Essai sur l'origine des connaissances humaines. P in e 1, Philippe: Traité médicophilosophique sur l'aliénation mentale. E s qu i r o l, Etienne: Les maladies mentales considérées sous le rapport médicale. $\mathrm{S} \mathrm{e} \mathrm{m} \mathrm{e} \mathrm{.}$ l a i g n e, René: Les grands aliénistes français. 1894. B u s q u e t, Paul: Les Biographies médicales. 1927/28. Bei diesem ausführliche Literaturangaben über Pinel und Esquirol. K 1 ö k h of , Cornelius Albert: De morbis animi ab infirmitate tenore medullae cerebri dissertatio. 1753. R e il, Johann Christian: Rapsodieen über die Anwendung der psych. Kurmethode auf Geisteszerrüttungen. 1818. C o n o 11 y , John: Die Behandlung der Irren ohne mechan. Zwang. 1860 deutsche Ausgabe. G ri e s i n g e r, Wilhelm: Gesammelte Abhandlungen. 1872. K r a e pe l i n, Emil: Hundert Jahre Psychiatrie. 1918. M o r s i e r, G. de: La neurologie à Genève. Médecine et Hygiène 1946. 